\title{
Calibration Risk for Exotic Options
}

\author{
Kai Detlefsen* \\ Wolfgang K. Härdle**
}

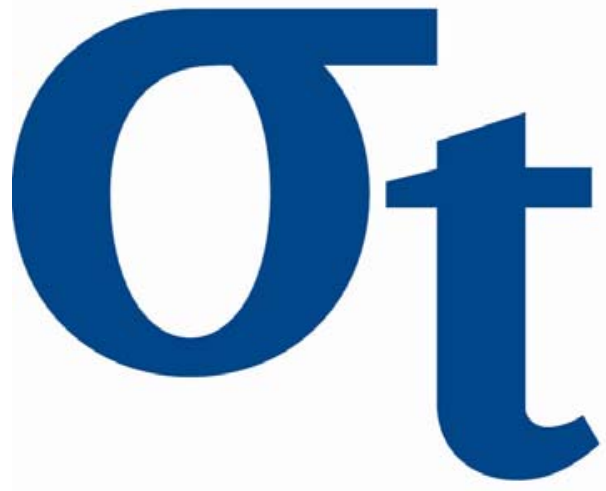

* CASE - Center for Applied Statistics and Economics,

Humboldt-Universität zu Berlin, Germany

This research was supported by the Deutsche Forschungsgemeinschaft through the SFB 649 "Economic Risk". 


\title{
Calibration Risk for Exotic Options
}

\author{
K. Detlefsen and W. K. Härdle \\ CASE - Center for Applied Statistics and Economics \\ Humboldt-Universität zu Berlin \\ Wirtschaftswissenschaftliche Fakultät \\ Spandauer Strasse 1, 10178 Berlin, Germany
}

\begin{abstract}
Option pricing models are calibrated to market data of plain vanillas by minimization of an error functional. From the economic viewpoint, there are several possibilities to measure the error between the market and the model. These different specifications of the error give rise to different sets of calibrated model parameters and the resulting prices of exotic options vary significantly. These price differences often exceed the usual profit margin of exotic options.

We provide evidence for this calibration risk in a time series of DAX implied volatility surfaces from April 2003 to March 2004. We analyze in the Heston and in the Bates model factors influencing these price differences of exotic options and finally recommend an error functional. Moreover, we determine the model risk of these two stochastic volatility models for the time series and consider its relation to calibration risk.
\end{abstract}

Key Words: calibration risk, calibration, model risk, Heston model, Bates model, barrier option, cliquet option

JEL Classification: C13, G12

Acknowledgement: This research was supported by Deutsche Forschungsgemeinschaft through the SFB 649 "Economic Risk" and by Bankhaus Sal. Oppenheim. The authors would like to thank Bernd Engelmann and Peter Schwendner for helpful discussions. 


\section{Introduction}

Recently, there has been a considerable interest, both from a practical and a theoretical point of view, in the risks involved in option pricing. Schoutens et al. (2004) have analyzed model risk in an empirical study and Cont (2004) has put this risk into a theoretical framework. Another source of risk is hidden in the calibration of models to market data. This calibration risk exceeds often the profit margin for exotic options and hence is also of fundamental interest for the banking industry. Moreover, calibration risk exists even if an appropriate model has been chosen and model risk does not exist anymore.

Calibration risk arises from the different possibilities to measure the error between the observations on the market and the corresponding quantities in the model world. A natural approach to specify this error is to consider the absolute price (AP) differences, see e.g. Schoutens et al. (2004). But the importance of absolute price differences depends on the magnitude of these price. Hence, another useful way for measuring the error are relative price (RP) differences, see e.g. Mikhailov et al. (2003). As models are often judged by their capability to reproduce implied volatility surfaces other measures can be defined in terms of implied volatilities. There are again the two possibilities of absolute implied volatilities (AI) and relative implied volatilities (RI). We consider these four ways to measure the difference between model and market data and explore the implications for the pricing of exotic options.

To this end, we focus on the popular stochastic volatility model of Heston. In order to analyze the influence of the goodness of fit on calibration risk we consider in addition the Bates model which is an extension of the Heston model with similar qualitative features. These two models are calibrated to plain vanillas on the DAX. In order to get reliable results we use a time series of implied volatility surfaces from April 2003 to March 2004. Because of the computationally intense Monte Carlo simulations for the pricing of the exotic options we consider only one trading day in each week of this period. As exotic options we consider down and out puts, up and out calls and cliquet options for 1, 2 or 3 years to maturity. In this framework we determine the size of calibration risk and analyze factors influencing it.

Besides calibration risk there is also model risk which represents wrong prices because a wrong parametric model has been chosen. We consider also model risk between the Heston and the Bates model and 
analyze the relation between the two forms of risk in pricing exotic options.

Section 2 introduces the models and describes their risk neutral dynamics that we use for option pricing. Moreover, this section contains information about the data used for the calibration. Section 3 describes the calibration method and defines the error functionals analyzed in this work. The goodness of fit is shown by representative surfaces and statistics on the errors. In Section 4 , we present the exotic options that we consider for calibration risk and price these products by simulation. In Section 5, we analyze the model risk for the two stochastic volatility models under the four error functionals. In the last Section 6, we summarize the results and draw our conclusions.

\section{Models and Data}

In this section, we describe briefly the Heston model and the Bates model for which we are going to analyze calibration risk. Moreover, we provide some descriptive statistics of the implied volatility surfaces that we use as input data for the calibration.

\subsection{Heston model}

We consider the popular stochastic volatility model of Heston (1993):

$$
\frac{d S_{t}}{S_{t}}=\mu d t+\sqrt{V_{t}} d W_{t}^{1}
$$

where the volatility process is modelled by a square-root process:

$$
d V_{t}=\xi\left(\eta-V_{t}\right) d t+\theta \sqrt{V_{t}} d W_{t}^{2}
$$

and $W^{1}$ and $W^{2}$ are Wiener processes with correlation $\rho$.

The volatility process remains positive if its volatility $\theta$ is small enough with respect to the product of the mean reversion speed $\xi$ and the average volatility level $\eta$ :

$$
\xi \eta>\frac{\theta^{2}}{2}
$$


The dynamics of the price process are analyzed under a martingale measure under which the characteristic function of $\log \left(S_{t}\right)$ is given by:

$$
\begin{aligned}
\phi_{t}^{H}(z)= & \exp \left\{\frac{-\left(z^{2}+\mathbf{i} z\right) V_{0}}{\gamma(z) \operatorname{coth} \frac{\gamma(z) t}{2}+\xi-\mathbf{i} \rho \theta z}\right\} \\
& \times \frac{\exp \left\{\frac{\xi \eta t(\xi-\mathbf{i} \rho \theta z)}{\theta^{2}}+\mathbf{i} z t r+\mathbf{i} z \log \left(S_{0}\right)\right\}}{\left(\cosh \frac{\gamma(z) t}{2}+\frac{\xi-\mathbf{i} \rho \theta z}{\gamma(z)} \sinh \frac{\gamma(z) t}{2}\right)^{\frac{2 \xi \eta}{\theta^{2}}}}
\end{aligned}
$$

where $\gamma(z) \stackrel{\text { def }}{=} \sqrt{\theta^{2}\left(z^{2}+\mathbf{i} z\right)+(\xi-\mathbf{i} \rho \theta z)^{2}}$, see e.g. Cont et al. (2004).

\subsection{Bates model}

Bates (1996) extended the Heston model by considering jumps in the stock price process:

$$
\begin{aligned}
\frac{d S_{t}}{S_{t}} & =\mu d t+\sqrt{V_{t}} d W_{t}^{1}+d Z_{t} \\
d V_{t} & =\xi\left(\eta-V_{t}\right) d t+\theta \sqrt{V_{t}} d W_{t}^{2}
\end{aligned}
$$

where $Z$ is a compound Poisson process with intensity $\lambda$ and jumps $k$ that have a lognormal distribution:

$$
\log (1+k) \sim \mathrm{N}\left(\log (1+\bar{k})-\frac{\delta^{2}}{2}, \delta^{2}\right) .
$$

We analyze the dynamics of this model under a martingale measure under which the characteristic function of $\log \left(S_{t}\right)$ is given by:

$$
\begin{aligned}
\phi_{t}^{B}(z)= & \exp \left\{t \lambda\left(e^{-\delta^{2} z^{2} / 2+\mathbf{i}\left\{\log (1+\bar{k})-\frac{1}{2} \delta^{2}\right\} z}-1\right)\right\} \\
& \times \exp \left\{\frac{-\left(z^{2}+\mathbf{i} z\right) V_{0}}{\gamma(z) \operatorname{coth} \frac{\gamma(z) t}{2}+\xi-\mathbf{i} \rho \theta z}\right\} \\
& \times \frac{\exp \left\{\frac{\xi \eta t(\xi-\mathbf{i} \rho \theta z)}{\theta^{2}}+\mathbf{i} z t(r-\bar{k})+\mathbf{i} z \log \left(S_{0}\right)\right\}}{\left(\cosh \frac{\gamma(z) t}{2}+\frac{\xi-\mathbf{i} \rho \theta z}{\gamma(z)} \sinh \frac{\gamma(z) t}{2}\right)^{\frac{2 \xi \eta}{\theta^{2}}}}
\end{aligned}
$$

where $\gamma(z) \stackrel{\text { def }}{=} \sqrt{\theta^{2}\left(z^{2}+\mathbf{i} z\right)+(\xi-\mathbf{i} \rho \theta z)^{2}}$, see e.g. Cont et al. (2004).

The Bates model has eight parameters while the Heston model has only five parameters. Because of these three additional parameters the Bates model can better fit observed surface but parameter stability is more difficult to achieve. 


\begin{tabular}{c|ccc}
\hline \hline & $\begin{array}{c}\text { mean number } \\
\text { of maturities }\end{array}$ & $\begin{array}{c}\text { mean number } \\
\text { of obervations }\end{array}$ & $\begin{array}{c}\text { mean money- } \\
\text { ness range }\end{array}$ \\
\hline $\begin{array}{c}\text { short maturities } \\
(0.25 \leq T<1.0) \\
\text { long maturities } \\
(1.0 \leq T)\end{array}$ & 3.06 & 64 & 0.553 \\
\hline total & 5.98 & 76 & 0.699 \\
\hline \hline
\end{tabular}

Table 1: Description of the implied volatility surfaces.

\section{$2.3 \quad$ Data}

Our data consists of EUREX-settlement volatilities of European options on the DAX. We consider the time period from April 2003 to March 2004. Since March 2003 the EUREX trades plain vanillas with maturities up to 5 years. Until March 2004 it has not changed its range of products. Hence, the data is homogeneous in sense that the implied volatility surfaces are derived from similar products.

From this time period we analyze the surfaces from all the Wednesdays when trading has taken place. Thus, we consider 51 implied volatility surfaces. We exclude observations that are deep out of the money because of illiquidity of these products. More precisely, we consider only options with moneyness $m=K / S_{0} \in[0.75,1.35]$ for small times to maturity $T \leq 1$. As we analyze exotic options with maturity in 1, 2 or 3 years we exclude also plain vanillas with time to maturity less than 3 months.

Some information about the resulting implied volatility surfaces are summarized in table 1. The surfaces contain in the mean 140 transformed prices and nine maturities with a mean moneyness range of $65 \%$.

The values of the underlying in the considered period are shown in figure 1. This figure contains also the (interpolated) implied volatilities for 1 year to maturity with strike at spot level. Hence, the market of the DAX went up in this period while the implied volatilities went down as figure 1 shows.

We approximate the risk free interest rates by the EURIBOR. On each trading day we use the yields corresponding to the maturities of the implied volatility surface. As the DAX is a performance index it 

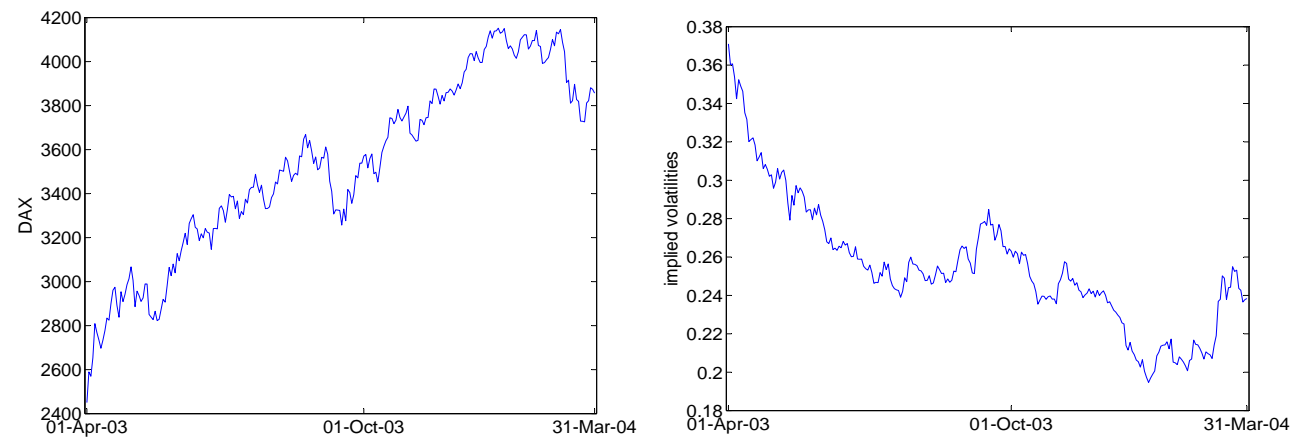

Figure 1: DAX and ATM implied volatility with 1 year to maturity on the trading days from 01 April 2003 to 31 March 2004.

is adjusted to dividend payments. Thus, we do not consider dividend payments explicitly.

\section{Calibration}

In this section, we specify the calibration routine and describe the four error functionals. The calibration results illustrate that the plain vanilla prices can be well replicated by the Heston and the Bates model.

\subsection{Calibration method}

Carr \& Madan (1999) found a representation of the price of a European call option by one integral for a whole class of option pricing models. Their method that is applicable to the Heston (1993) model is based on the characteristic function of the log stock price under the risk neutral measure.

Carr and Madan showed that the price $C(K, T)$ of a European call option with strike $K$ and maturity $T$ is given by

$$
C(K, T)=\frac{\exp \{-\alpha \ln (K)\}}{\pi} \int_{0}^{+\infty} \exp \{-\mathbf{i} v \ln (K)\} \psi_{T}(v) d v
$$

for a (suitable) damping factor $\alpha>0$. The function $\psi_{T}$ is given by

$$
\psi_{T}(v)=\frac{\exp (-r T) \phi_{T}\{v-(\alpha+1) \mathbf{i}\}}{\alpha^{2}+\alpha-v^{2}+\mathbf{i}(2 \alpha+1) v}
$$


where $\phi_{T}$ is the characteristic function of $\log \left(S_{T}\right)$, see Section 2 .

For the minimization we consider the following four objective functions based on the root weighted square error:

$$
\begin{aligned}
& \mathrm{AP} \stackrel{\text { def }}{=} \sqrt{\sum_{i=1}^{n} w_{i}\left(P_{i}^{\text {mod }}-P_{i}^{\text {mar }}\right)^{2}} \\
& \mathrm{RP} \stackrel{\text { def }}{=} \sqrt{\sum_{i=1}^{n} w_{i}\left(\frac{P_{i}^{\text {mod }}-P_{i}^{\text {mar }}}{P_{i}^{\text {mar }}}\right)^{2}} \\
& \mathrm{AI} \stackrel{\text { def }}{=} \sqrt{\sum_{i=1}^{n} w_{i}\left(I V_{i}^{\text {mod }}-I V_{i}^{\text {mar }}\right)^{2}} \\
& \mathrm{RI} \stackrel{\text { def }}{=} \sqrt{\sum_{i=1}^{n} w_{i}\left(\frac{I V_{i}^{\text {mod }}-I V_{i}^{\text {mar }}}{I V_{i}^{\text {mar }}}\right)^{2}}
\end{aligned}
$$

where mod refers to a model quantity and mar to a quantity observed on the market, $P$ to a price and $I V$ to an implied volatility. The index $i$ runs over all $n_{t}$ observations of the surface on day $t$. The weights $w_{i}$ are non negative with $\sum_{i} w_{i}=1$. Hence, the objective functions can be interpreted as mean average errors.

We choose the weights in such a way that on each day all maturities have the same influence on the objective function. In order to make different surfaces comparable each maturity gets the weight $1 / n_{\text {mat }}$ where $n_{\text {mat }}$ denotes the number of maturities in this surface. Moreover, we assign the same weight to all points of the same maturity. This leads to the weights

$$
w_{i} \stackrel{\text { def }}{=} \frac{1}{n_{m a t} n_{s t r}^{i}}
$$

where $n_{s t r}^{i}$ denotes the number of strikes with the same maturity as observation $i$. This weighting leads asymptotically to a uniform density on each maturity.

Given these weights we measure the average time to maturity of an implied volatility surface by a modified duration:

$$
\sum_{i=1}^{n} \frac{\tau_{i} w_{i}}{\sum_{i=1}^{n} w_{i}}
$$


where $\tau_{i}$ is the time to maturity of the option $i$. The mean duration of the 51 surfaces is 2.02 and the minimal (maximal) is 1.70 (2.30). Thus, the point of balance for the maturities lies around 2 for our time series of surfaces. As we analyze exotic options with 1, 2 or 3 years time to maturity this point of balance confirms a correct weighting for our purposes.

As prices we consider only out of the money prices. Thus, we use call prices for strikes higher (or equal) than the spot and put prices for strikes below the spot. This approach ensures to compare only prices of similar magnitude. It has no impact on the errors based on implied volatilities. Because of the put call parity the use of OTM options has nor an impact on the absolute price error (AP). But the relative prices are weighted in such a way that the observations around the spot receive less weight. Hence, only the relative price error (RP) is influenced by this choice of prices.

In order to estimate the model parameters we apply a stochastic global optimization routine and minimize the objective functions with respect to the model parameters. In addition to some natural constraints on the range of the parameters we have taken into account inequality 1 that ensures the positivity of the volatility process.

Sometimes the objective function that is minimized contains in addition to the error measure a regularization term. Regularization can be necessary for two reasons: The error function may have several global minima and thus the regularization is necessary in order to get a unique minimum. Besides this static problem it is also important to find parameters on subsequent days that lead to similar prices (and greeks) of exotic options. This time stability is essential for the practical applicability of the calibration. We have discovered in tests on simulated and real data that our algorithm finds a unique solution whatever the starting conditions are. As we do not consider subsequent trading days in our analysis the time stability is not essential in our case. Hence we omit a regularization term.

\subsection{Calibration results}

We have considered the implied volatility surfaces of each Wednesday in the period from April 2003 to March 2004 on which trading has taken place. Thus, we have analyzed 51 surfaces. Each of these has been calibrated with respect to the four error functions described in Section 3.1. These calibrations have been done for the Heston and the 


\begin{tabular}{|c|c|c|c|c|}
\hline $\begin{array}{l}\text { mean } \\
\text { objective fct. }\end{array}$ & $\mathrm{AP}$ & $\begin{array}{c}\mathrm{RP} \\
{\left[E^{-2}\right]}\end{array}$ & $\begin{array}{c}\mathrm{AI} \\
{\left[E^{-2}\right]}\end{array}$ & $\begin{array}{c}\mathrm{RI} \\
{\left[E^{-2}\right]}\end{array}$ \\
\hline $\mathrm{AP}$ & 7.3 & 9.7 & 0.81 & 3.1 \\
\hline $\mathrm{RP}$ & 11. & 6.1 & 0.74 & 2.9 \\
\hline AI & 9.4 & 7.3 & 0.68 & 2.6 \\
\hline RI & 8.8 & 7.0 & 0.70 & 2.5 \\
\hline
\end{tabular}

Table 2: Calibration errors in the Heston model for 51 days.

\section{Bates model.}

The resulting errors of these 408 calibrations have been summarized in table 2 for the Heston model and in table 3 for the Bates model. Descriptive statistics on the calibrated parameters are given in the appendix in table 9 for the Heston model and in table 10 for the Bates model. Figure 2 shows the fit of the implied volatility surface in the Heston model on a day that is representative for the AI error.

Table 2 reports in each line the means of the four errors when the objective function given in the left column is minimized. In the Heston model, we get a mean absolute price error of 7.3 and a mean relative price error of $9.7 \%$ when we calibrate with respect to AP. Using the $\mathrm{RP}$ error functional we get the opposite result with a mean absolute price error of 11 and a mean relative price error of $6.1 \%$. The errors based on implied volatilities are smaller for the RP objective function than for the AP objective function. The results for the AI and RI objective functionals differ only slightly: the mean absolute implied volatility error is about $0.68 \%$ and the mean relative implied volatility error is about $2.5 \%$. Moreover, the price errors for these objective functions lie between the price errors of the other two objective functions. The calibration w.r.t. RI gives the best overall fit because it has the smallest RI error and the second best errors for the rest. The meaning of these error measures is illustrated by figure 2 which shows an implied volatility fit that is representative for an AI error of $0.68 \%$. In order to make the AP errors comparable for different days (with different values of the spot) we have computed the mean of AP/DAX as $0.21,0.34,0.27,0.25$ for the four error functionals.

The calibrated parameters which are described in the appendix by table 9 form two groups because the parameters for the RP, AI and RI calibration are quite similar. The start volatility $V_{0}$ and the average volatility level $\eta$ are both about $7 \%$ for all objective functionals. For 


\begin{tabular}{cc|cccc}
\hline \hline & mean & AP & $\begin{array}{c}\text { RP } \\
{\left[E^{-2}\right]}\end{array}$ & $\begin{array}{c}\text { AI } \\
{\left[E^{-2}\right]}\end{array}$ & $\begin{array}{c}\text { RI } \\
{\left[E^{-2}\right]}\end{array}$ \\
\hline objective fct. & & 7.0 & 13. & 0.76 & 2.8 \\
AP & 12. & 5.1 & 0.67 & 2.6 \\
RP & & 8.9 & 6.4 & 0.60 & 2.3 \\
AI & & 8.7 & 6.2 & 0.62 & 2.2 \\
RI & &
\end{tabular}

Table 3: Calibration errors in the Bates model for 51 days.

the AP calibration we get a reversion speed $\xi=0.9$, a volatility of volatility of $\theta=0.34$ and a correlation $\rho=-0.82$. The other calibrations lead to similar parameters with a reversion speed $\xi=1.3$, a volatility of volatility of $\theta=0.44$ and a correlation $\rho=-0.75$. As the correlations are significantly below -1 the calibrated Heston models have really two stochastic factors.

The Bates model exhibits similar qualitative results as the Heston model: The AP and the RP calibrations differ widely while the AI and the RI calibrations lead to similar results. The Bates model can regarded as an extension of the Heston model. The additional three parameters for the jumps in the spot process lead for all errors functionals to better calibration results: The AP error is reduced (in the mean) by $4 \%, \mathrm{RP}$ error by $16 \%$, the AI and the RI error both by $12 \%$.

The calibrated parameters of the Bates model are given in table 10 . As in the Heston model they form two groups with the AP calibration on the one hand and the RP, AI and RI calibrations on the other hand. The parameters $\xi, \eta, \theta$ and $V_{0}$ are similar to the calibrations for the Heston model. Only the correlation $\rho$ rises to a level of -0.93 for all objective functions. Hence, this criterion for distinguishing between the two groups disappears. It is replaced by the expected number of jumps per year: For the AP calibration we expect (in the mean) a jump every three years while we expect every two years a jump for the other calibrations. It is interesting that all calibrations lead to a mean jump up of about $+8 \%$ for the returns. The expected jumps upwards correspond to the market going up as shown in figure 1 .

Schoutens et al. (2004) found that the Heston and the Bates option model can both be calibrated well to the EuroStoxx50. In summarizing the results of this section we can say that also DAX implied volatility surfaces can be replicated well by these models for different error functionals. As Schoutens et al. (2004), we find that the Bates 

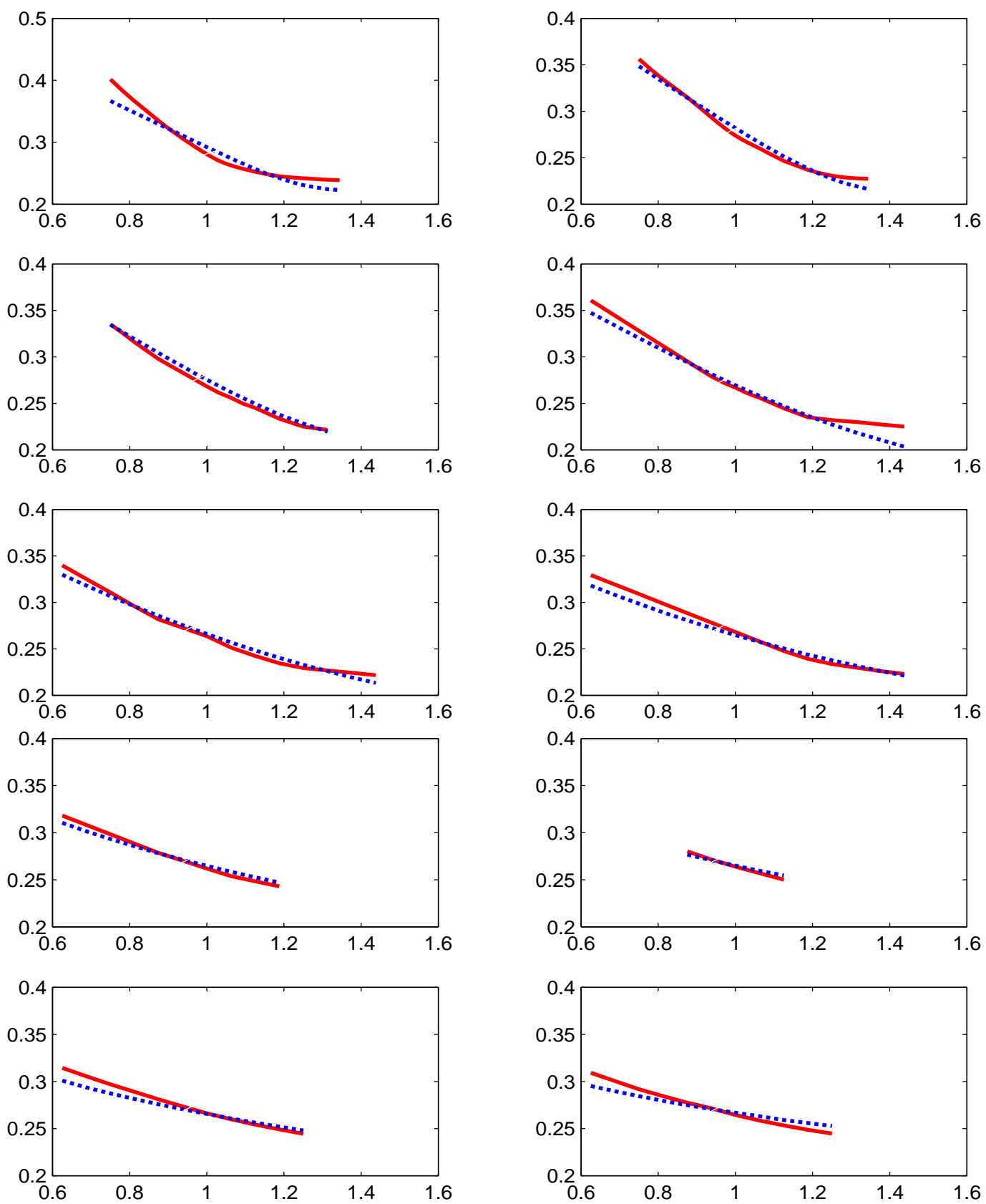

Figure 2: Implied volatilities in the Heston model for the maturities 0.26, $0.52,0.78,1.04,1.56,2.08,2.60,3.12,3.64,4.70$ (left to right, top to bottom) for AI parameters on 25/6/2003. Red solid: model, blue dotted: market. $\mathrm{X}$-axis: moneyness. 
model gives only slightly better fits for the AP calibration. In addition we have shown that it leads to a considerable improvement in the fit for the other objective functions.

\section{Exotic Options}

We come now to the analysis of the price differences of exotic options for calibrations w.r.t. different error measures. We consider barrier and cliquet options. The prices of these products are calculated by Monte Carlo simulations using Euler discretizations.

\subsection{Simulation}

We price all exotic options by Monte Carlo simulations. To this end, we use for each derivate product 1000000 paths generated by Euler discretization, see e.g. Glasserman (2004). For each exotic option we consider three maturities: 1 year, 2 years and 3 years. We analyze three exotic options: up \& out calls, down \& out puts and cliquet options. These products are described in the following sections where remaining parameters are also specified.

The payoffs of barrier options depend on the minimum or maximum of the underlying price process in some time interval. We approximate this quantity by a discrete minimum with one observation for each trading day. Thus, we use 250 time steps to simulate a process for a year.

The calibration results are presented in following sections together with a discussion of the options. The accuracy of the Monte Carlo results is given by the relative standard error in table 4. Thus, this table confirms that the estimators have sufficiently small variance after 1000000 paths compared to the price differences we observe in tables 5 to 7 .

\subsection{Barrier Options}

For the barrier options that are very popular on the market we consider two types: up \& out calls and down \& out puts. 


\begin{tabular}{c|ccc|ccc}
\hline \hline & \multicolumn{3}{|c|}{ Heston } & \multicolumn{3}{c}{ Bates } \\
& $T=1$ & $T=2$ & $T=3$ & $T=1$ & $T=2$ & $T=3$ \\
\hline up and out calls & 0.17 & 0.10 & 0.08 & 0.17 & 0.11 & 0.09 \\
down and out puts & 0.18 & 0.11 & 0.08 & 0.19 & 0.12 & 0.10 \\
cliquet options & 0.06 & 0.05 & 0.05 & 0.07 & 0.06 & 0.05 \\
\hline \hline
\end{tabular}

Table 4: Maximal relative standard error in percent of Monte Carlo simulations. (Maximum over all time points and all objective functions)

\subsubsection{Up and out call options}

The prices of up and out calls with strike $K$, barrier $B$ and maturity $T$ on an underlying $\left(S_{t}\right)$ are given by

$$
\exp (-r T) \mathrm{E}\left[\left(S_{T}-K\right)^{+} \mathbf{1}_{\left\{M_{T}<B\right\}}\right]
$$

where

$$
M_{T} \stackrel{\text { def }}{=} \max _{0 \leq t \leq T} S_{t}
$$

We choose as strike $K$ and barrier $B$

$$
\begin{aligned}
K & =1-0.1 T \\
B & =1+0.2 T
\end{aligned}
$$

where $T$ denotes time to maturity. Up and out calls with such strikes and barriers are widely traded on the market.

Up and out call options have the payoff profile of European call options if the underlying has not fallen below the barrier. Otherwise their payoff is zero. Thus, up and out calls are path dependent exotic options.

We want to analyze the difference between the prices of the exotic options when the underlying model has been calibrated w.r.t. different errors. To this end, we have calibrated the Heston and the Bates model to implied volatility or price data on each day w.r.t. the four error functionals introduced in Section 3.1. Hence we have four time series of calibrated model parameters that are described in Section 3.2. By Monte Carlo simulations we calculate on each day the prices of up and out calls for the four sets of model parameters. In this way we get four 


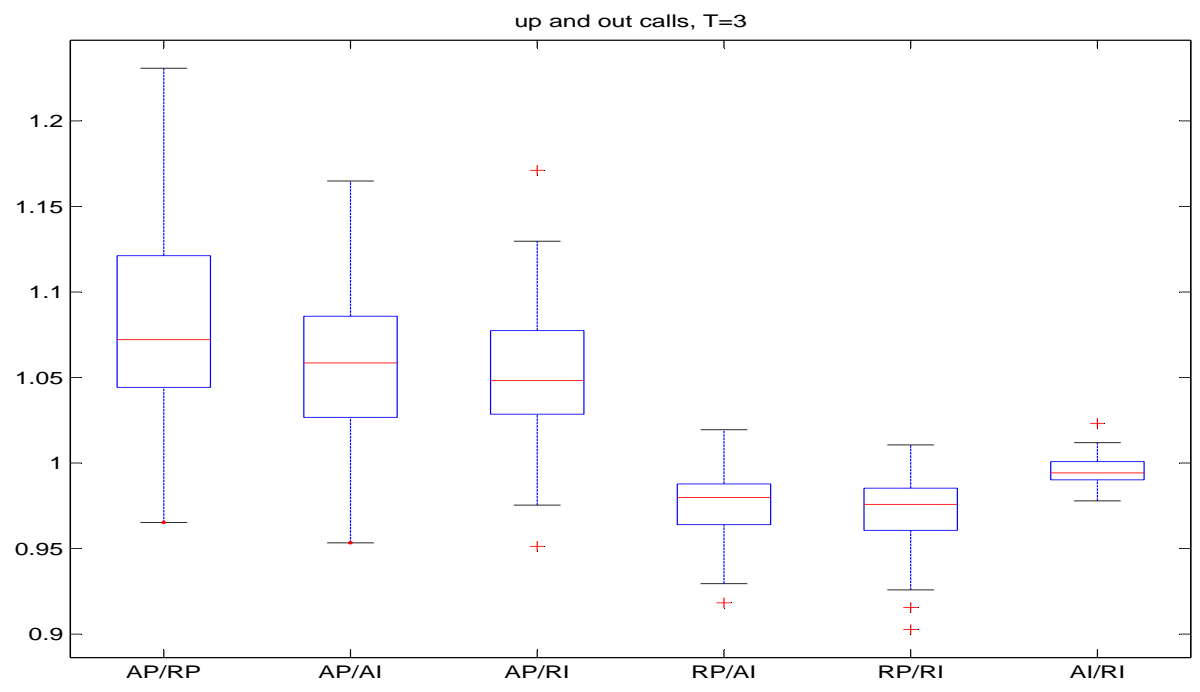

Figure 3: Relative prices of the up and out calls in the Heston model for 3 years to maturity.

time series of up and out call prices corresponding to the four error measures. As we are interested how the price varies over the four error functionals we consider the quotients of the up and out call prices.

The six possible time series of price quotients are shown in figure 3 for 3 years to maturity in the Heston model. In the boxplots the central red line gives the median and the box contains $50 \%$ of the observations. Hence, the AP prices lie in the mean about $6 \%$ over the other prices and the AP prices are in $75 \%$ of the 51 days at least $4 \%$ higher than the other prices. The RP prices are about $2 \%$ below the AI or RI prices which are very similar to each other.

We analyze the influence of time to maturity on these price differences by considering also 1 year and 2 years to maturity (and by adjusting the barrier and the strike appropriately). The medians of the price quotients are presented in table 5 for all three times to maturity. This table shows that the price differences become smaller for shorter times to maturity for the AP prices. The other price differences remain almost constant. For 1 year to maturity the price differences are about $2 \%-3 \%$ and the AP prices are lower than the other prices. For 2 years to maturity the AP prices are again higher than the other prices. 


\begin{tabular}{cc|cccccc}
\hline \hline & & $\mathrm{AP} / \mathrm{RP}$ & $\mathrm{AP} / \mathrm{AI}$ & $\mathrm{AP} / \mathrm{RI}$ & $\mathrm{RP} / \mathrm{AI}$ & $\mathrm{RP} / \mathrm{RI}$ & $\mathrm{AI} / \mathrm{RI}$ \\
\hline Heston & $T=1$ & 0.986 & 0.968 & 0.967 & 0.984 & 0.984 & 0.999 \\
& $T=2$ & 1.051 & 1.024 & 1.022 & 0.979 & 0.978 & 0.998 \\
& $T=3$ & 1.072 & 1.059 & 1.048 & 0.980 & 0.976 & 0.994 \\
\hline Bates & $T=1$ & 0.988 & 0.985 & 1.002 & 1.002 & 1.006 & 1.012 \\
& $T=2$ & 1.070 & 1.083 & 1.104 & 0.970 & 0.986 & 1.018 \\
& $T=3$ & 1.106 & 1.123 & 1.129 & 0.972 & 0.975 & 1.013 \\
\hline \hline
\end{tabular}

Table 5: Median of price quotients of up and out calls.

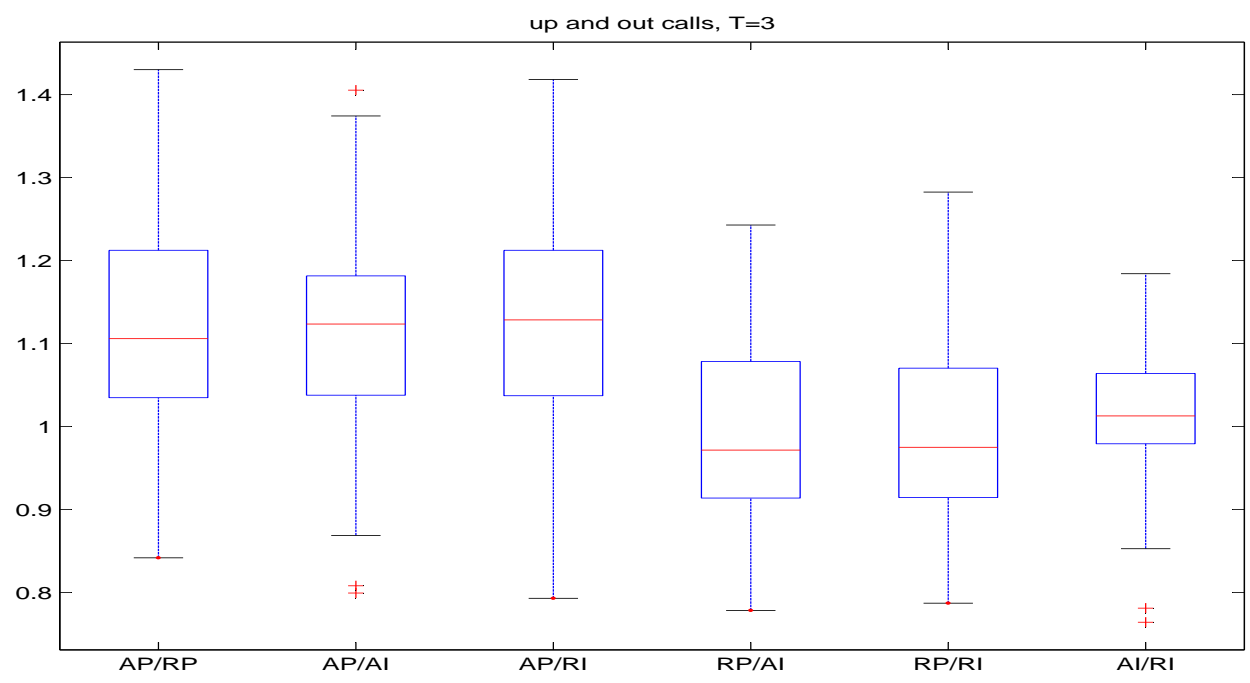

Figure 4: Relative prices of the up and out calls in the Bates model for 3 years to maturity. 
In order to analyze the influence of the goodness of fit on the price differences we consider also the Bates model. The boxplots of the price quotients in this model are given in figure 4 for 3 years to maturity. Compared to the Heston boxplots the boxes are longer in the Bates model. Thus there is more variation between the prices for different error functionals. Moreover the median differences between the AP prices and the other prices are bigger than in the Heston model - especially for AP/AI and AP/RI. The differences between RP, AI and RI are similar to those in the Heston model. The corresponding results for 1 year and 2 years to maturity are presented in table 5. Quantitatively the situation is similar to the Heston model: For shorter times to maturity the price differences decrease - especially for AP prices.

Thus, the price differences in the Heston and in the Bates model are similar between the RP, AI and RI prices while the AP price differences are bigger in the Bates model. Moreover, the variation of the price differences is higher in the Bates model.

\subsubsection{Down and out put options}

The prices of the down and out puts with strike $K$, barrier $B$ and maturity $T$ on an underlying $\left(S_{t}\right)$ are given by

$$
\exp (-r T) \mathrm{E}\left[\left(S_{T}-K\right)^{+} \mathbf{1}_{\left\{m_{T}>B\right\}}\right]
$$

where

$$
m_{T} \stackrel{\text { def }}{=} \min _{0 \leq t \leq T} S_{t}
$$

For our analysis, we use the strike $K$ and the barrier $B$

$$
\begin{aligned}
K & =1+0.1 T \\
B & =1-0.2 T
\end{aligned}
$$

where $T$ denotes time to maturity. The strikes and barriers are set in analogy to the up and out calls. Such down and out puts are again a typical product on the exotics markets.

Down and out put options have the payoff profile of European put options if the underlying has been below the barrier during the life time of the option. Otherwise their payoff is zero.

As described above, we calculate on each day the prices of the down and out puts for the four parameter sets. The resulting six time series 


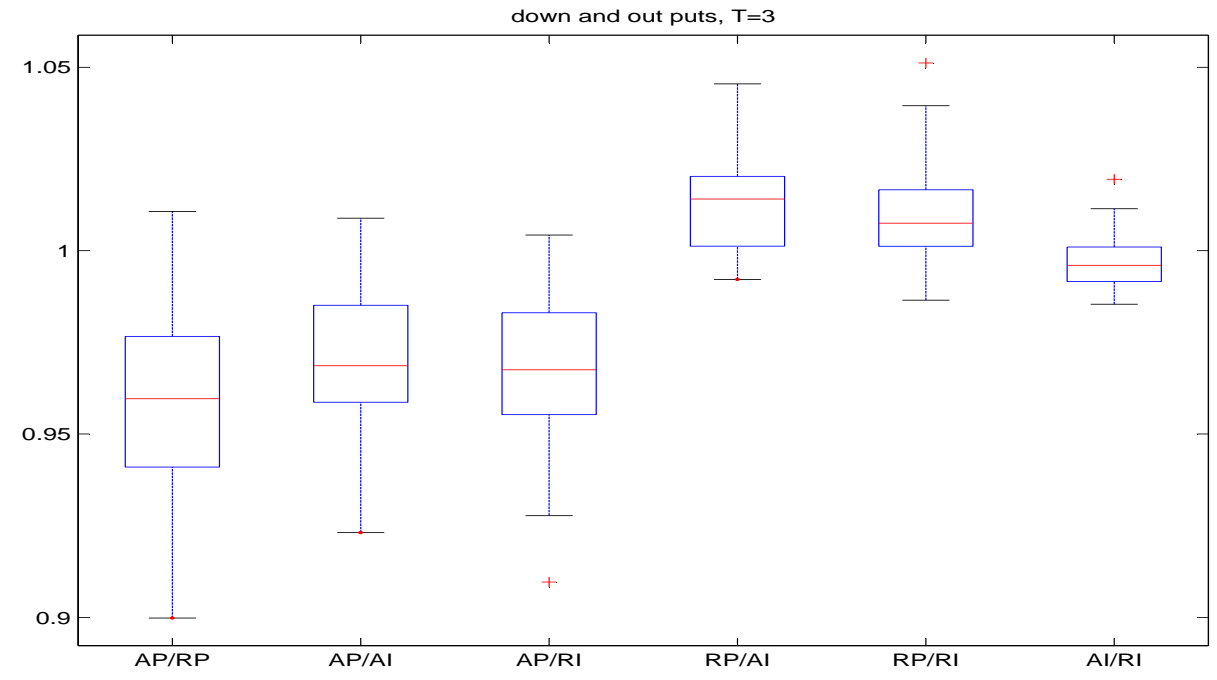

Figure 5: Relative prices of the down and out puts in the Heston model for 3 years to maturity.

of price quotients are shown in the figure 5 for 3 years to maturity in the Heston model. The AP prices are (in the mean) about 3.5\% smaller than the other prices and $75 \%$ of the AP prices are at least $2 \%$ smaller than the other prices. The RP prices lie above the prices from the calibrations to implied volatilities. These AI and RI prices are quite similar so that we can identify again the two groups that we have already observed for the up and out calls.

Compared to the up and out calls the price differences are smaller for the down and out puts. This can be seen also from table 6 that reports the median of the price quotients for 1,2 and 3 years to maturity. This table shows that the price differences change for increasing time to maturity: For 1 year to maturity the AP prices lie above the other prices but with increasing time to maturity the AP prices become relatively smaller. The RP and AI prices remain on a comparable level for all times to maturity and the RI prices tend to this level for longer times to maturity.

The situation in the Bates model that gives better fits to the plain vanilla data is given by figure 6 and table 6 . The AP prices lie about $7 \%$ below the other prices. Thus this difference is bigger than in the Heston model. The other price quotient lie still in the mean on the 


\begin{tabular}{cc|cccccc}
\hline \hline & & $\mathrm{AP} / \mathrm{RP}$ & $\mathrm{AP} / \mathrm{AI}$ & $\mathrm{AP} / \mathrm{RI}$ & $\mathrm{RP} / \mathrm{AI}$ & $\mathrm{RP} / \mathrm{RI}$ & $\mathrm{AI} / \mathrm{RI}$ \\
\hline Heston & $T=1$ & 1.025 & 1.031 & 1.005 & 1.007 & 0.980 & 0.977 \\
& $T=2$ & 0.983 & 0.994 & 0.984 & 1.011 & 0.997 & 0.986 \\
& $T=3$ & 0.960 & 0.969 & 0.968 & 1.014 & 1.008 & 0.996 \\
\hline \multirow{3}{*}{ Bates } & $T=1$ & 1.021 & 1.012 & 1.019 & 1.004 & 1.006 & 0.998 \\
& $T=2$ & 0.968 & 0.975 & 0.966 & 1.031 & 1.022 & 0.990 \\
& $T=3$ & 0.922 & 0.935 & 0.931 & 1.026 & 1.022 & 0.995 \\
\hline \hline
\end{tabular}

Table 6: Median of price quotients of down and out puts.

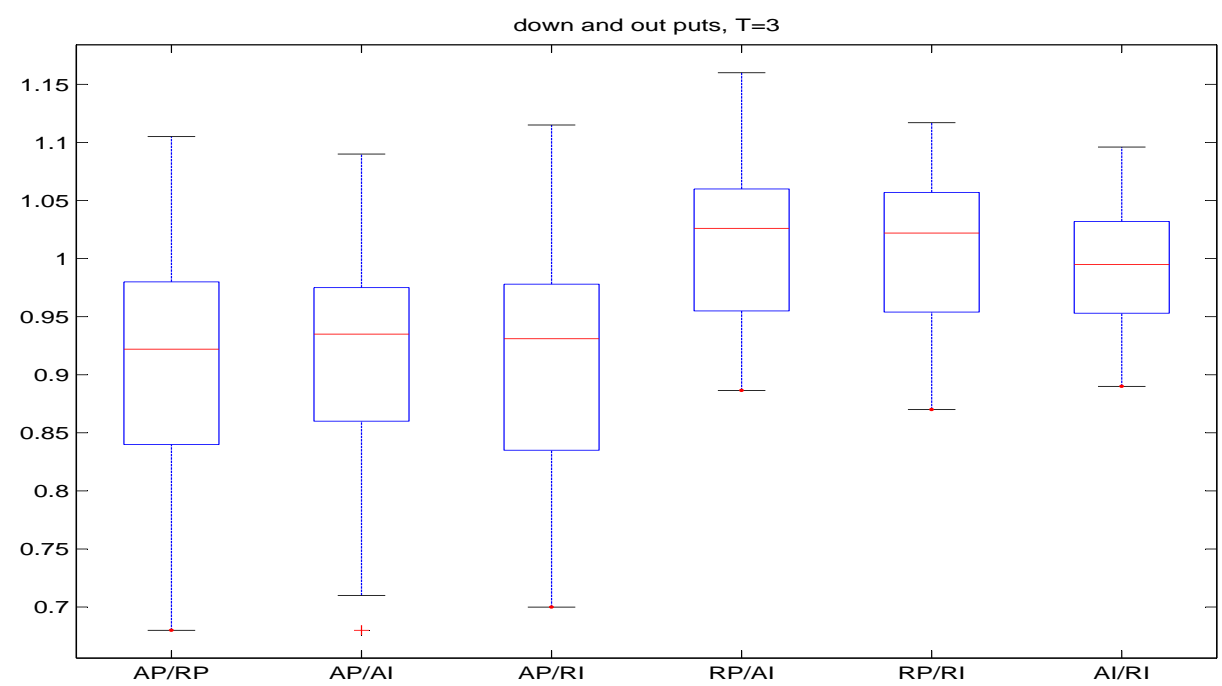

Figure 6: Relative prices of the down and out puts in the Bates model for 3 years to maturity. 
same level but the their variance has grown compared to the Heston model.

The situation for the barrier options can be summarized as follows: The AP prices differ significantly from the other prices for both barrier options. While the AP prices are higher for up and out calls they are lower for down and out puts relatively to the other prices. In this sense the situation is symmetrical. The differences become bigger for longer times to maturity and the better fit of the Bates model does not lead to smaller price differences.

\subsection{Cliquet Options}

We consider cliquet options with prices

$$
\exp (-r T) \mathrm{E}[H]
$$

where the payoff $H$ is given by

$$
H \stackrel{\text { def }}{=} \min \left(c_{g}, \max \left[f_{g}, \sum_{i=1}^{N} \min \left\{c_{l}^{i}, \max \left(f_{l}^{i}, \frac{S_{t_{i}}-S_{t_{i-1}}}{S_{t_{i-1}}}\right)\right\}\right]\right) .
$$

Here $c_{g}\left(f_{g}\right)$ is a global cap (floor) and $c_{g}^{i}\left(f_{g}^{i}\right)$ is a local cap (floor) for the period $\left[t_{i-1}, t_{i}\right]$.

We consider three periods with $t_{i}=\frac{T}{3} i(i=0, \ldots, 3)$ and the caps and floors are given by

$$
\begin{aligned}
c_{g} & =\infty \\
f_{g} & =0 \\
c_{l}^{i} & =0.08, \quad i=1,2,3 \\
f_{l}^{i} & =-0.08, \quad i=1,2,3
\end{aligned}
$$

Cliquet options have many parameters. Hence, this specification cannot give representative picture of all the traded cliquets. But these caps and floors are typical because the option holder cannot loose money and the returns is bounded above only by the local return bounds.

Cliquet options pay out basically the sum of the returns $R_{i} \stackrel{\text { def }}{=}$ $\frac{S_{t_{i}}-S_{t_{i-1}}}{S_{t_{i-1}}}$. In order to reduce risk local and global floors $f$ are introduced for the returns $R$. In the same way the returns are bounded above by local and global caps $c$. 
The distributions of the six time series of price quotients for cliquet options are described in figure 7 for 3 years to maturity in the Heston model. The differences are smaller than in the case of the barrier options. The AP prices lie above the other prices but the difference is significant only for the AP and RP prices. The differences between the other prices is also small. Thus, we cannot recognize directly from this figure the two groups that we identified for the barrier options.

Table 7 that reports the median price differences for 1,2 and 3 years to maturity gives some insight into this situation: The AP prices are about $2 \%$ smaller than the other prices for 1 year to maturity. With increasing time to maturity the AP prices grow relatively and are about $1.5 \%$ higher than the other prices for 3 years to maturity. As table 7 confirms the other prices remain relatively constant for different times to maturity. Thus there are again the two groups that we have identified for the barrier options: The changing AP prices on the one hand and the constant other prices on the other hand.

The relative prices of the cliquet options in the Bates model are presented in figure 8 for 3 years to maturity. Here we see that the AP prices are about $7 \%$ smaller than the other prices. The RP prices lie about $2 \%$ under the AI prices that are $3 \%$ higher than the RI prices. The RP and RI prices are similar. Thus, there are quite big differences for the cliquet options in the Bates model. Moreover, the variance is larger relative to the Heston model. Table 7 describes the situation of different times to maturity and shows that the AP prices grow relatively with increasing time to maturity while the other prices remain relatively constant for different times to maturity.

Comparing the results for the two barrier options and the cliquets we see in all cases two groups, the AP prices and the other prices. The AP prices differ a lot from the other prices and in addition change relatively for different times to maturity. Moreover, the variance of the price quotient with AP prices is bigger in general than for the other price quotients. The other group of RP, AI and RI prices shows similar prices and small variances. The Bates model that gives better fits has higher price differences (with higher variances). 


\begin{tabular}{cc|cccccc}
\hline \hline & & $\mathrm{AP} / \mathrm{RP}$ & $\mathrm{AP} / \mathrm{AI}$ & $\mathrm{AP} / \mathrm{RI}$ & $\mathrm{RP} / \mathrm{AI}$ & $\mathrm{RP} / \mathrm{RI}$ & $\mathrm{AI} / \mathrm{RI}$ \\
\hline Heston & $T=1$ & 0.983 & 0.976 & 0.989 & 0.993 & 1.006 & 1.013 \\
& $T=2$ & 1.002 & 0.991 & 1.000 & 0.989 & 0.998 & 1.010 \\
& $T=3$ & 1.022 & 1.008 & 1.014 & 0.987 & 0.992 & 1.005 \\
\hline \multirow{4}{*}{ Bates } & $T=1$ & 0.917 & 0.899 & 0.917 & 0.987 & 1.005 & 1.024 \\
& $T=2$ & 0.931 & 0.903 & 0.923 & 0.980 & 0.999 & 1.029 \\
& $T=3$ & 0.946 & 0.912 & 0.933 & 0.976 & 0.995 & 1.029 \\
\hline \hline
\end{tabular}

Table 7: Median of price quotients of cliquet options.

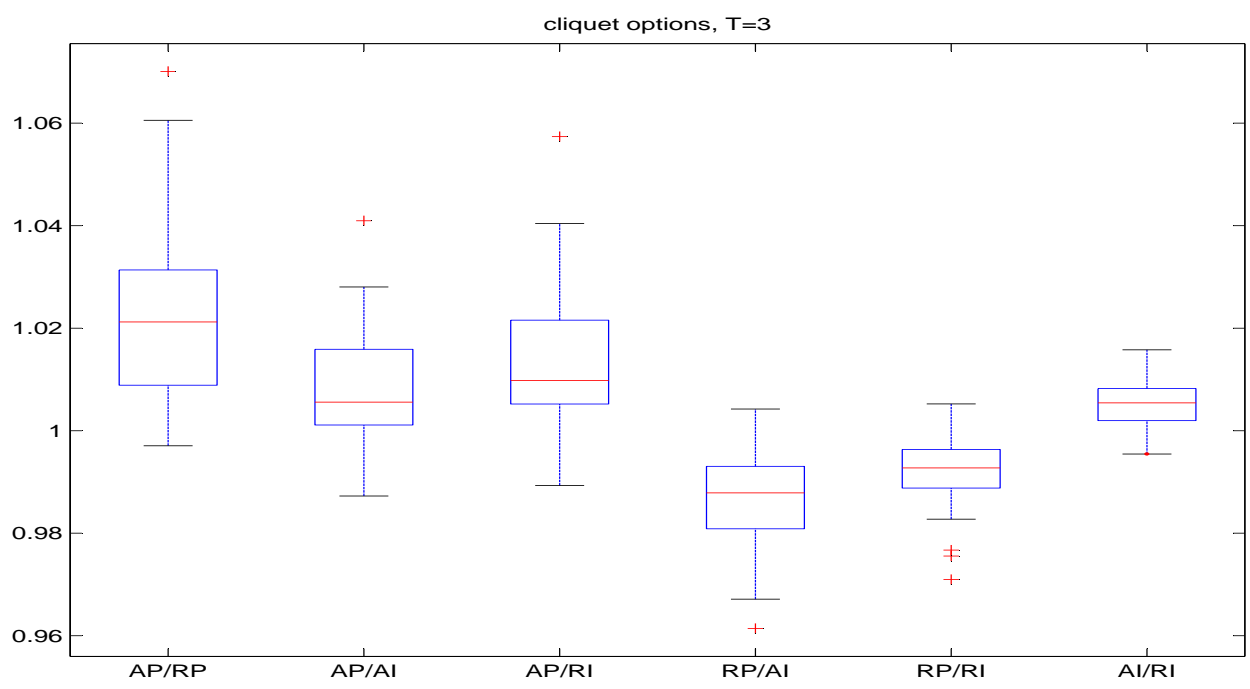

Figure 7: Relative prices of the cliquet options in the Heston model for 3 years to maturity. 


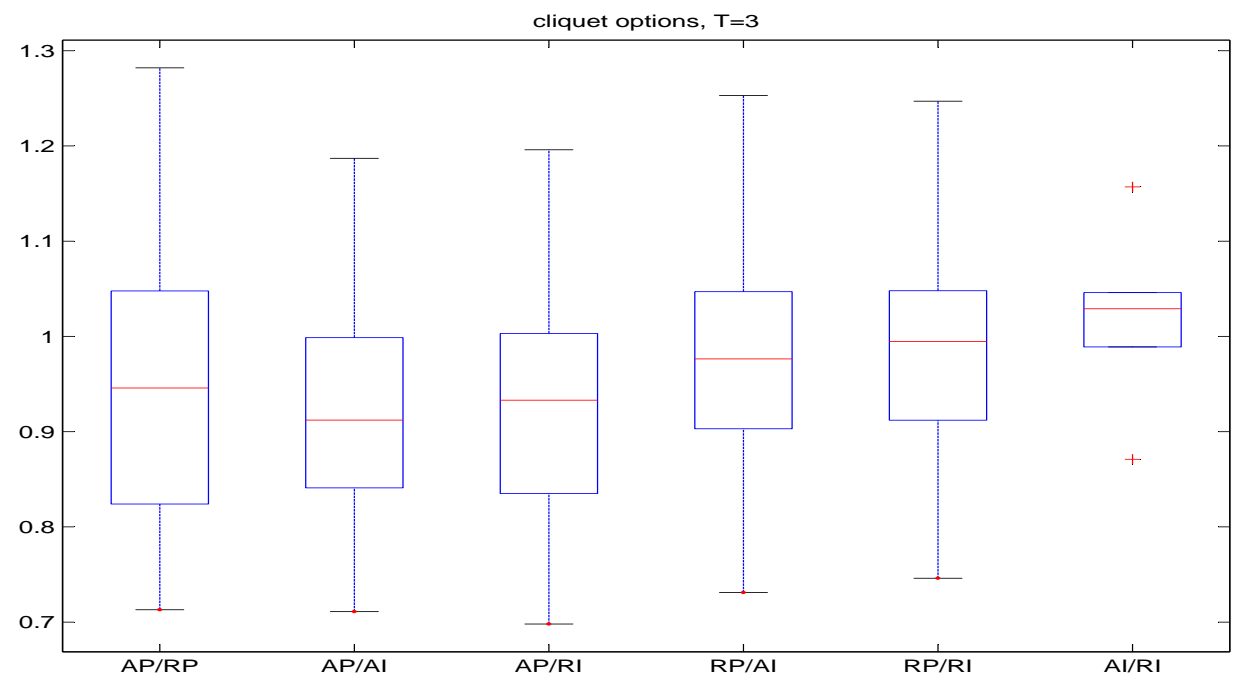

Figure 8: Relative prices of the cliquet options in the Bates model for 3 years to maturity.

\section{Model risk}

In the last section, we have described the price differences that result from the calibration w.r.t. the four error functionals. In this section we consider model risk, consider its relation to calibration risk and compare our results with the findings of Schoutens et al. (2004). Model risk is generally understood as the risk of wrong prices because a wrong parametric model has been chosen for the stochastic process of the underlying.

In order to analyze this model risk for the two stochastic volatility models, we consider the quotients of the prices of the exotic options in the Bates model and the corresponding prices in the Heston model. The distribution of these quotients for up and out calls with 3 years to maturity is described by the figure 9. The prices in the Bates model lie below the prices in the Heston model for all four error functionals: The difference varies between $2 \%$ for the AP prices and $6 \%$ for the RI prices. Thus model risk is not independent of the calibration method, i.e. calibration risk. The results for smaller times to maturity are given in table 8. The table suggests that model risk does not change significantly for different times to maturity. 


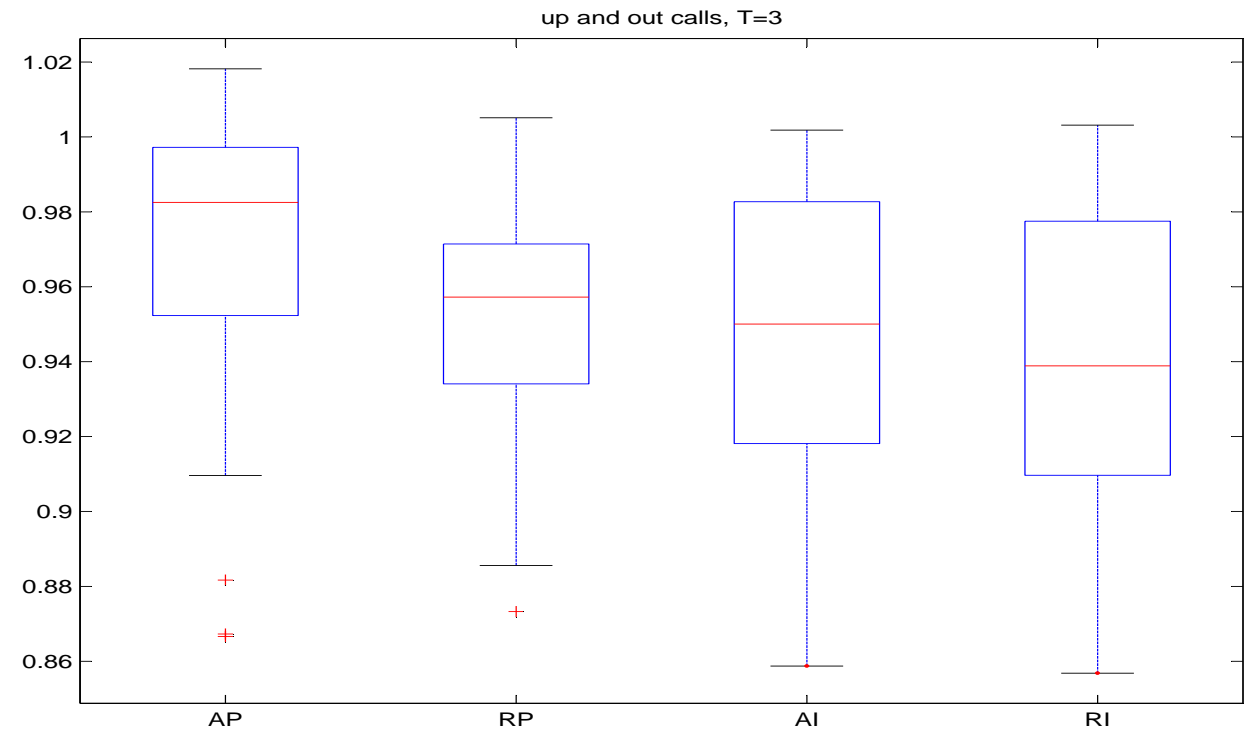

Figure 9: Bates prices over Heston prices for up and out calls with 3 years to maturity on 51 days.

\begin{tabular}{cc|cccc}
\hline \hline & & AP & RP & AI & RI \\
\hline up and out calls & $T=1$ & 0.973 & 0.953 & 0.944 & 0.941 \\
& $T=2$ & 0.980 & 0.954 & 0.953 & 0.940 \\
& $T=3$ & 0.983 & 0.957 & 0.950 & 0.939 \\
\hline down and out puts & $T=1$ & 0.933 & 0.892 & 0.877 & 0.878 \\
& $T=2$ & 0.918 & 0.883 & 0.872 & 0.860 \\
& $T=3$ & 0.916 & 0.881 & 0.873 & 0.860 \\
\hline cliquets & $T=1$ & 1.057 & 1.100 & 1.109 & 1.119 \\
& $T=2$ & 1.076 & 1.128 & 1.130 & 1.144 \\
& $T=3$ & 1.086 & 1.138 & 1.140 & 1.162 \\
\hline \hline
\end{tabular}

Table 8: Median of Bates prices over Heston prices. 


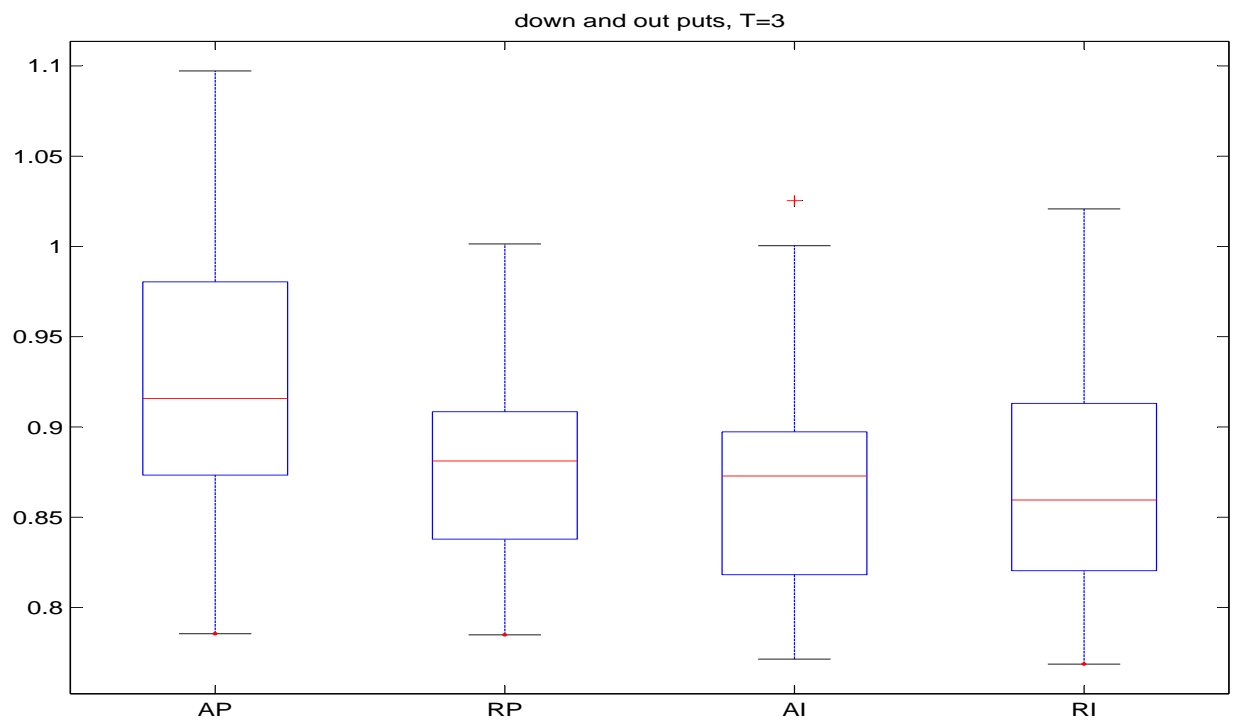

Figure 10: Bates prices over Heston prices for down and out puts with 3 years to maturity on 51 days.

The model risk of down and out puts is shown in figure 10 for 3 years to maturity. The prices in the Bates model lie below the prices in the Heston model for all error functionals. Compared to the up and out calls the model risk is bigger for the down and out puts: It varies between $9 \%$ for AP prices and $14 \%$ for RI prices. But again we observe the highest difference for RI prices and the smallest for AP prices. Moreover, the variance is bigger than for the up and out calls. Table 8 that gives the results for smaller times to maturity suggests that the model risk becomes smaller for shorter times to maturity.

Finally, we consider the model risk of cliquet options in figure 11 . For these options the Bates prices lie above the corresponding Heston prices for all calibration methods. The smallest price difference that appears for the AP prices is about $8 \%$ while the biggest difference of $16 \%$ have the RI prices. Table 8 shows again smaller price differences for shorter times to maturity.

The model risk between the Heston and the Bates model can be described for barrier and cliquet options as follows: Model risk measured by the price differences in the two models increasing for longer times 


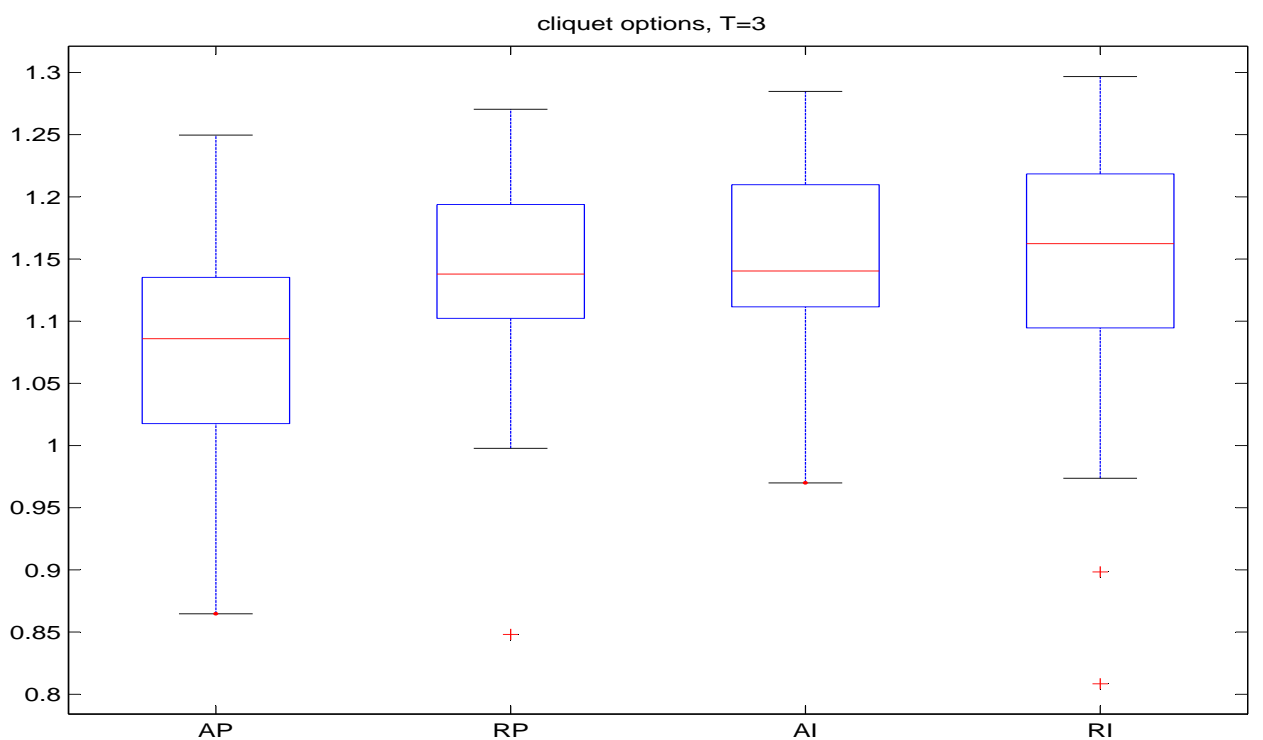

Figure 11: Bates prices over Heston prices for cliquet options with 3 years to maturity on 51 days.

to maturity. Moreover, it is ordered w.r.t. the calibration method. The calibration w.r.t. implied volatilities leads to bigger price differences as calibration w.r.t. prices. The model risk is smallest for AP calibration and bigger for RP calibration. It is even bigger for the AI calibration and the price differences are the biggest for RI calibrations. This emphasizes once more the importance of the implied volatility surfaces and their calibration. Moreover, model risk differs across option types.

Schoutens et al. (2004) consider up and out calls (with strike equal to spot) and cliquet options with 3 years to maturity. For a barrier $50 \%$ above the spot, they find a model risk for the up and out calls of about 14\%. For the cliquet options Schoutens et al. do not find a significant model risk. These results do not correspond in every respect to our AP results. There may be several reasons for these different results: While we look at a time series of 51 implied volatility surfaces they focus one one day. They have analyzed the EuroStoxx50 and we use DAX data. 


\section{Conclusion}

We have looked at the popular stochastic volatility model of Heston and analyzed different calibration methods and their impact on the pricing of exotic options. Our analysis was carried out for a time series of DAX implied volatility surfaces from April 2003 to March 2004.

We have shown that different ways to measure the error between the model and the market in the calibration routine lead to significant price differences of exotic options in the sense that these differences often exceed the profit margins of the products. We have considered the four error measures that are defined by the root mean squared error of absolute or relative differences of prices or implied volatilities. Among these measures we have identified two groups: Calibrations w.r.t. relative prices, absolute implied volatilities or relative implied volatilities lead to similar prices of exotic options. Calibrations w.r.t. absolute prices imply exotics prices that are quite different from the prices of the first group. The price differences increase for longer times to maturity. Moreover, the differences do not decrease in the Bates model although it is an extension of the Heston model with similar qualitative features and a better fit to plain vanilla data. The price differences of exotic options differ also across option types and are bigger for barrier options than for cliquets.

Moreover, we have looked at the model risk for these two option pricing models. Model risk and calibration risk are not independent because model risk is lowest for calibrations w.r.t. absolute prices and highest for calibrations w.r.t. relative implied volatilities. As this holds for all considered options model risk seems to be ordered w.r.t. the error measure used in the calibration.

As model risk is bigger than calibration risk calibrations should be carried out w.r.t. absolute prices if the choice of an appropriate model is unclear. But if a model has already been chosen we suggest to measure the error between the model and the market in terms of (relative) implied volatilities because this error measure reflects best the characteristics of the model that are essential for exotic options. Moreover, we have demonstrated that this choice leads to good calibrations (e.g. relatively good fits and stable parameters). We have

also shown that the resulting prices of exotic options often lie in the middle of the prices from the other calibrations and have the smallest variance. Our results underline the importance of the implied volatility surface and suggest that one should measure the error in the 
calibration in terms of implied volatilities.

\section{References}

Bates, D. (1996). Jump and Stochastic Volatility: Exchange Rate Processes Implicit in Deutsche Mark Options, Review of Financial Studies 9: 69-107.

Carr, P. \& Madan, D. (1999). Option valuation using the fast Fourier transform, Journal of Computational Finance 2: 61-73.

Cont, R. (2005 ). Model uncertainty and its impact on the pricing of derivative instruments, to appear in Mathematical Finance.

Cont, R. \& Tankov, P. (2004). Financial Modelling With Jump Processes, Chapman \& Hall/CRC.

Glasserman, P. (2004). Monte Carlo Methods in Financial Engineering, Springer, New York.

Heston, S. (1993). A closed-form solution for options with stochastic volatility with applications to bond and currency options, Review of Financial Studies 6: 327-343.

Mikhailov, S. \& Nögel, U. (2003). Heston's stochastic volatility model. Implementation, calibration and some extensions, Wilmott magazine, July 2003.

Schoutens, W., Simons, E. \& Tistaert, J. (2004). A Perfect Calibration! Now What? Wilmott magazine, March 2004. 


\begin{tabular}{c|ccccc}
\hline \hline & $\xi$ & $\eta$ & $\theta$ & $\rho$ & $V_{0}$ \\
\hline AP & 0.87 & 0.07 & 0.34 & -0.82 & 0.07 \\
& $(0.48)$ & $(0.02)$ & $(0.08)$ & $(0.08)$ & $(0.02)$ \\
RP & 1.38 & 0.07 & 0.44 & -0.74 & 0.08 \\
& $(0.35)$ & $(0.02)$ & $(0.06)$ & $(0.03)$ & $(0.02)$ \\
AI & 1.32 & 0.07 & 0.43 & -0.77 & 0.08 \\
& $(0.40)$ & $(0.02)$ & $(0.06)$ & $(0.04)$ & $(0.02)$ \\
RI & 1.20 & 0.07 & 0.41 & -0.75 & 0.08 \\
& $(0.35)$ & $(0.02)$ & $(0.06)$ & $(0.05)$ & $(0.02)$ \\
\hline \hline
\end{tabular}

Table 9: Mean parameters (std.) in the Heston model for 51 days.

\begin{tabular}{c|cccccccc}
\hline \hline & $\xi$ & $\eta$ & $\theta$ & $\rho$ & $V_{0}$ & $\lambda$ & $\bar{k}$ & $\delta$ \\
\hline AP & 0.92 & 0.07 & 0.33 & -0.94 & 0.07 & 0.33 & 0.07 & 0.08 \\
& $(0.50)$ & $(0.02)$ & $(0.08)$ & $(0.07)$ & $(0.02)$ & $(0.21)$ & $(0.03)$ & $(0.06)$ \\
RP & 1.56 & 0.07 & 0.45 & -0.89 & 0.08 & 0.54 & 0.05 & 0.08 \\
& $(0.47)$ & $(0.02)$ & $(0.07)$ & $(0.07)$ & $(0.02)$ & $(0.23)$ & $(0.03)$ & $(0.06)$ \\
AI I & 1.43 & 0.07 & 0.43 & -0.95 & 0.07 & 0.50 & 0.06 & 0.09 \\
& $(0.44)$ & $(0.02)$ & $(0.06)$ & $(0.06)$ & $(0.02)$ & $(0.22)$ & $(0.03)$ & $(0.04)$ \\
RI & 1.36 & 0.07 & 0.41 & -0.93 & 0.07 & 0.52 & 0.05 & 0.08 \\
& $(0.44)$ & $(0.02)$ & $(0.07)$ & $(0.09)$ & $(0.02)$ & $(0.26)$ & $(0.04)$ & $(0.08)$ \\
\hline \hline
\end{tabular}

Table 10: Mean parameters (std.) in the Bates model for 51 days. 


\section{SFB 649 Discussion Paper Series 2006}

For a complete list of Discussion Papers published by the SFB 649, please visit http://sfb649. wiwi. hu-berlin.de.

001 "Calibration Risk for Exotic Options" by Kai Detlefsen and Wolfgang K. Härdle, January 2006.

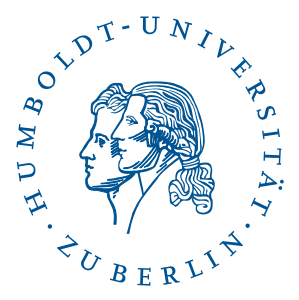

